\title{
Robust Coarsening in Multiscale PDEs
}

Robert Scheich ${ }^{1}$

\section{Introduction}

Consider a variationally-posed 2nd-order elliptic boundary value problem

$$
a(u, v) \equiv \int_{\Omega} \mathcal{A}(\mathbf{x}) \nabla u \cdot \nabla v=\int_{\Omega} f(\mathbf{x}) v(\mathbf{x}), \quad \text { for all } v \in H_{0}^{1}(\Omega),
$$

with solution $u \in H_{0}^{1}(\Omega)$ and domain $\Omega \subset \mathbb{R}^{d}, d=2,3$, where the coefficient tensor $\mathcal{A}(\mathbf{x})$ is highly heterogeneous (possibly in a spatially complicated way). We assume that $\mathcal{A}(\mathbf{x})$ is symmetric, uniformly positive definite and mildly anisotropic, i.e. $\lambda_{\min }(\mathcal{A}(\mathbf{x})) \gtrsim \lambda_{\max }(\mathcal{A}(\mathbf{x}))$ uniformly in $\mathbf{x}$. We are particularly interested in the case when the contrast $\max _{\mathbf{x}, \mathbf{y} \in \Omega} \lambda_{\max }(\mathcal{A}(\mathbf{x})) / \lambda_{\max }(\mathcal{A}(\mathbf{y}))$ is large. Many examples of this type arise in subsurface flow modelling or in material science. The space $H_{0}^{1}(\Omega)$ is the usual Sobolev space of functions with vanishing trace on $\partial \Omega$ and $f \in H^{-1}(\Omega)$. For simplicity we assume for the remainder that $\mathcal{A}(\mathbf{x})=\alpha(\mathbf{x}) I$, i.e. a scalar diffusion coefficient.

Let $\mathcal{T}_{h}$ be a simplicial triangulation of $\Omega$ and let (1) be discretised in $V_{h} \subset H_{0}^{1}(\Omega)$, the space of continuous, piecewise linear FE functions with respect to $\mathcal{T}_{h}$ that vanish on $\partial \Omega$. For simplicity let $\mathcal{T}_{h}$ be quasi-uniform. The $a$-orthogonal projection of $u$ to $V_{h}$ is denoted by $u_{h}$. In the usual nodal basis $\left\{\varphi_{i}\right\}_{i=1}^{n}$ for $V_{h}$, the problem of finding $u_{h}$ reduces to the $n \times n$ linear system

$$
A \mathbf{u}=\mathbf{b}
$$

with stiffness matrix $A=\left(a\left(\varphi_{i}, \varphi_{j}\right)\right)_{i, j=1}^{n}$. Since the matrix $A$ depends on $\alpha$ only through element averages, we can assume (w.l.o.g.) that $\alpha$ is piecewise constant with respect to $\mathcal{T}_{h}$. For simplicity we assume that $\alpha$ is piecewise constant with respect to some non-overlapping partitioning of $\Omega$ into open, connected Lipschitz polyhedra (polygons) $\left\{\mathcal{Y}_{m}\right\}_{m=1}^{M}$ and set $\alpha_{m}=\left.\alpha\right|_{\mathcal{Y}_{m}}$.

Dept. Mathematical Sciences, University of Bath, Bath, UK. R.Scheichl@bath.ac.uk 
Especially for $d=3$ and for problems where $\alpha$ varies on a small length scale $\varepsilon \ll \operatorname{diam}(\Omega)$, and thus the mesh size $h$ needs to be very fine, multilevel iterative solvers (multigrid, domain decomposition, etc) are usually essential to solve this problem efficiently. Their scalability and robustness with respect to mesh refinement, as well as other discretisation parameters has been studied extensively. Here we will focus on their robustness with respect to coefficient variation. We will show that coefficient robustness is inherently linked to a judicious choice of coarse space $V_{H}$ (related to some coarse mesh $\mathcal{T}_{H}$ with resolution $H$ ). If $\varepsilon \gtrsim H$ and if we can choose a coarse mesh such that all coefficient jumps are aligned with the mesh, then the coefficient robustness of standard coarse spaces has been analysed in the 90s (cf. $[4,3,10,16,25,21,22]$ and the references therein). For certain methods the robustness may depend on the quasi-monotonicity of the coefficient with respect to the coarse mesh (in the sense of [3]). Substructuring-type ("exotic") coarse spaces are usually used to achieve uniform coefficient robustness. A certain amount of robustness can be recovered for standard piecewise linear coarse spaces by using the multilevel solver as a preconditioner within CG (e.g. [24]). The key tool in all these analyses is the weighted $L_{2}$-projection of Bramble and $\mathrm{Xu}$ [1]. It requires a piecewise constant weight with respect to the coarse mesh, an assumption that is often far too stringent in real applications. We want to move away from this and crucially here make no assumptions that the underlying coarse grids resolve the coefficients.

A lot of effort in the last 25 years has gone into the development of algebraic methods to construct coarse spaces, such as algebraic multigrid (AMG), rather than analytic/geometric ones. It has been confirmed numerically that AMG methods are in practice robust to coefficient variation when applied to (2) (i.e. the number of iterations is unaffected), and they are therefore extremely popular. However, they are built on several heuristics and so a rigorous analysis of their coefficient-robustness is difficult (see [22] for a review of existing theoretical results). Nevertheless, the key principle of these algebraic coarse spaces, namely energy minimisation [11], also underlies many other coarse spaces. To obtain rigorous coefficient-independent convergence results we will need to work in the following energy and weighted $L_{2}$-norms on $D \subset \Omega$,

$$
\|v\|_{a, D}=\int_{D} \alpha|\nabla v|^{2} \quad \text { and } \quad\|v\|_{0, \alpha, D}=\int_{D} \alpha v^{2},
$$

respectively. When $D=\Omega$ we will usually not specify the domain explicitly.

A convenient framework to analyse most multilevel methods is the Schwarz or subspace correction framework [21, 23]. We restrict attention to the twolevel overlapping additive Schwarz method and focus on the robustness of various coarse spaces for this method. We review some recent papers on the topic mainly by the author (jointly with co-workers), as well as by Efendiev et al. All the results apply immediately also to multiplicative, hybrid and non-overlapping versions of the Schwarz method (see $[9,18]$ for some explicit comments). Many of the results can be extended to a multilevel theory $[18,5]$. 


\section{Schwarz framework and abstract coarse spaces}

Let us assume that $\left\{\Omega_{k}\right\}_{k=1}^{K}$ is an overlapping partitioning of $\Omega$ and let $\Omega_{k}^{\circ}$ be the overlap of subdomain $\Omega_{k}$, i.e. the set of points $\mathbf{x} \in \Omega_{k}$ that are contained in at least one other subdomain. We assume that $\mathcal{T}_{h}$ is aligned with this partitioning. Furthermore, let $\left\{\chi_{k}\right\}_{k=1}^{K} \subset V_{h}$ be an arbitrary partition of unity (POU) of FE functions subordinate to $\left\{\Omega_{k}\right\}_{k=1}^{K}$ such that $\left\|\chi_{k}\right\|_{\infty} \lesssim 1$ and $\left\|\nabla \chi_{k}\right\|_{\infty} \leq \delta_{k}^{-1}$, for all $k=1, \ldots, K$. Note that (due to quasi-uniformity of $\mathcal{T}_{h}$ ) we always have $\delta_{k} \gtrsim h$, and there is a partition of unity such that $\delta_{k}$ is proportional to the (minimal) width of $\Omega_{k}^{\circ}$. We assume as usual that each point $\mathbf{x} \in \Omega$ is contained in at most $N_{0}$ subdomains (finite covering).

We associate with each $\Omega_{k}$ the space $V_{k}=\left\{v \in V_{h}: \operatorname{supp}(v) \subset \bar{\Omega}_{k}\right\}$ and assume that we have an additional coarse space

$$
V_{0}=V_{H}=\operatorname{span}\left\{\Phi_{j} \in V_{h}: j=1, \ldots, N\right\} \subset V_{h}
$$

Let $\omega_{j}=$ interior $\left(\operatorname{supp}\left(\Phi_{j}\right)\right)$ and set $H_{j}=\operatorname{diam}\left(\omega_{j}\right)$. Then $H=\max _{j} H_{j}$ is the coarse mesh size associated with $V_{H}$.

The two-level additive Schwarz preconditioner is now simply

$$
M_{\mathrm{AS}}^{-1}=R_{0}^{T} A_{0}^{-1} R_{0}+\sum_{k=1}^{K} R_{k}^{T} A_{k}^{-1} R_{k} \quad \text { with } \quad A_{k}=R_{k} A R_{k}^{T} .
$$

$R_{k}$ is the matrix representation of a restriction operator from $V$ to $V_{k}$ : the simple injection operator for $k \geq 1$, and for $k=0$ induced by the coarse space basis $\left\{\Phi_{j}\right\}_{j=1}^{N}$ so that the coarse space stiffness matrix is $A_{0}=\left(a\left(\Phi_{j}, \Phi_{\ell}\right)\right)_{j, \ell}^{N}$.

The following result can be proved in the same way as [18, Thm. 2.5]. Since it is instructive, we give an outline of the proof.

Theorem 1. If there exists an operator $\Pi: V_{h} \rightarrow V_{0}$ such that for all $v \in V_{h}$

$$
\|\Pi v\|_{a}^{2} \leq C_{1}\|v\|_{a}^{2} \quad \text { and } \quad \sum_{k=1}^{K}\left\|(v-\Pi v) \nabla \chi_{k}\right\|_{0, \alpha}^{2} \leq C_{2}\|v\|_{a}^{2},
$$

then $\kappa\left(M_{\mathrm{AS}}^{-1} A\right) \lesssim C_{1}+C_{2}$. The hidden constant depends on $N_{0}$.

Proof. Let $v_{0}=\Pi v$ be such that (3) holds and choose $v_{k}=I_{h}\left(\chi_{k}\left(v-v_{0}\right)\right)$, where $I_{h}$ is the standard nodal interpolant on $V_{h}$. This interpolant is stable for all piecewise quadratic functions in the energy norm and in the weighted $L_{2}$-norm (independently of $\alpha$ ) (cf. [18, Lem. 2.3]), and so we get

$$
\begin{aligned}
\sum_{k=0}^{K}\left\|v_{k}\right\|_{a}^{2} & \lesssim\left\|v_{0}\right\|_{a}^{2}+\sum_{k=1}^{K}\left\|\chi_{k}\left(v-v_{0}\right)\right\|_{a}^{2} \\
& \lesssim\left\|v_{0}\right\|_{a}^{2}+\sum_{k=1}^{K}\left\|\chi_{k}\right\|_{\infty}^{2}\left\|v-v_{0}\right\|_{a, \Omega_{k}}^{2}+\left\|\left(v-v_{0}\right) \nabla \chi_{k}\right\|_{0, \alpha}^{2}
\end{aligned}
$$

Now, the boundedness of the POU functions, the finite cover assumption, as well as (3) lead to the stability estimate $\sum_{k=0}^{K}\left\|v_{k}\right\|_{a}^{2} \lesssim\left(C_{1}+C_{2}\right)\|v\|_{a}^{2}$. Since $v=\sum_{k=0}^{K} v_{k}$, the result follows from the abstract Schwarz theory (cf. [21]). 
This result shows the importance of the choice of coarse space. Provided we have a good coarse space approximation in the weighted $L_{2}$-norm that is moreover stable in the energy norm, independently of variations in $\alpha$, then the bound on the condition number for two-level additive Schwarz is also robust with respect to these variations. Note that it is crucial to use the weighted $L_{2}$ and the energy norm here to achieve coefficient-robustness, and that we only require weak $L_{2}$-approximation in regions where $\nabla \chi_{k} \neq 0$.

Several approaches have been studied in $[2,5,6,7,8,9,17,18,19]$ to provide constants in (3) that are independent of $\alpha$ (or at least of the contrast in $\alpha$ ) for various coarse spaces. However, in most cases the constants are not independent of $\frac{H}{\varepsilon}$, where $\varepsilon$ is the minimal length scale at which $\alpha$ varies in the regions where $\nabla \chi_{k} \neq 0$. So unfortunately in general, to be also independent of $\frac{H}{\varepsilon}$, restrictions on the coarse mesh size are needed, at least locally.

Let us discuss the assumptions (3) a bit further. Let $\Pi v=\sum_{j} f_{j}(v) \Phi_{j}$, where $f_{j}: V_{h} \rightarrow \mathbb{R}$ is a suitable functional. Then

$$
\|\Pi v\|_{a}=\left\|\sum_{j} f_{j}(v) \Phi_{j}\right\|_{a} \leq \sum_{j} \mid f_{j}(v)\left\|\Phi_{j}\right\|_{a} .
$$

We see that a set of coarse basis functions with bounded energy (independent of $\alpha$ ) is beneficial. The first approaches in $[8,9,17]$ attacked this target directly and aimed at bounding $\left\|\Phi_{j}\right\|_{a}$. In that case, it suffices to use the standard quasi-interpolant. Alternatively, a weighted quasi-interpolant with $f_{j}(v)=\int_{\omega_{j}} \alpha v / \int_{\omega_{j}} \alpha$ can be used. For certain (locally quasi-monotone) coefficients $\alpha$ this leads to a constant $C_{1}$ that is independent of the contrast in $\alpha$, even if the energy of the basis functions is not bounded (see below).

Similar comments can be made about the second assumption in (3). Note that

$$
\left\|(v-\Pi v) \nabla \chi_{k}\right\|_{0, \alpha}^{2} \leq\left\{\begin{array}{l}
\left\|\alpha\left|\nabla \chi_{k}\right|^{2}\right\|_{\infty}\|v-\Pi v\|_{0, \Omega_{k}^{\circ}}^{2}, \quad \text { or } \\
\left\|\nabla \chi_{k}\right\|_{\infty}^{2}\|v-\Pi v\|_{0, \alpha, \Omega_{k}^{\circ}}^{2} .
\end{array}\right.
$$

We can either try to choose a partition of unity $\left\{\chi_{k}\right\}$ such that $\left\|\alpha\left|\nabla \chi_{k}\right|^{2}\right\|_{\infty}$ is bounded independently of $\alpha$, which is again related to energy minimisation, or we can try to bound $\|v-\Pi v\|_{0, \alpha, \Omega_{k}^{\circ}}$ directly. As above, it is possible for certain (locally quasi-monotone) coefficients to achieve this and to obtain a constant $C_{2}$ that does not depend on the contrast in $\alpha$ (see below).

When the coefficient is not locally quasi-monotone, then it is in general necessary to enrich the coarse space, by either refining the coarse mesh locally, or by choosing more than one basis function per subdomain $\Omega_{k}$, with the key tool to achieve coarse space robustness being again energy minimisation.

To highlight some of the key issues we will use a number of representative model problems shown in Figure 1. For the rest of the paper, we will only focus on cases, such as Figures 1(c-h), where it is impossible or impractical that the subdomains $\left\{\Omega_{k}\right\}$ and the supports $\left\{\omega_{j}\right\}$ of the coarse basis functions resolve the coefficient jumps. The resolved cases in Figures $1(\mathrm{a}-\mathrm{b})$ have already been studied extensively, see e.g. [4, 3, 10, 16, 25, 21, 22, 24]. 


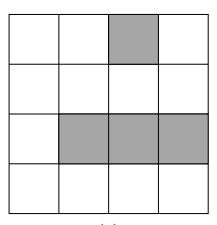

(a)

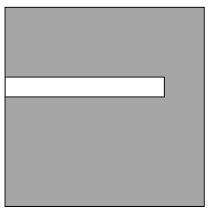

(e)

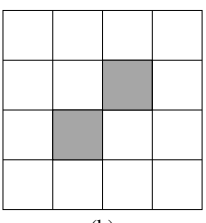

(b)

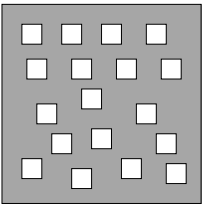

(f)

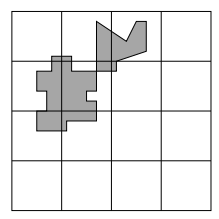

(c)

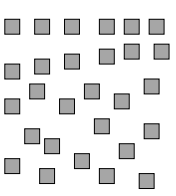

(g)

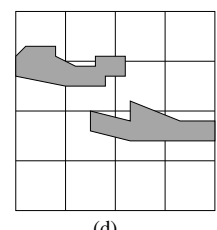

(d)

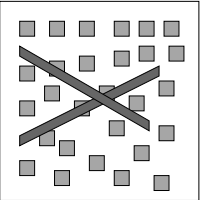

(h)

Fig. 1 Typical coefficient distributions (a) resolved; (b) not quasi-monotone; (c) neither quasi-monotone nor resolved; (d) channelised; (e) flow barriers; (f) low permeability inclusions; (g) high permeability inclusions; (h) high permeability inclusions and channels.

\section{Analysis of coefficient-robustness}

We present three possible approaches to try and prove coefficient robustness rigorously and thus to design robust coarse spaces. For simplicity, we assume that for each $j=1, \ldots, N$, there exists a $k=1, \ldots, K$ such that $\omega_{j} \subset \Omega_{k}$.

\subsection{Standard quasi-interpolant $\mathcal{E}$ energy minimisation}

The first approach makes use of the standard quasi-interpolant

$$
\Pi v=\sum_{j=1}^{N} \bar{v}_{\omega_{j}} \Phi_{j}, \quad \text { where } \quad \bar{v}_{\omega_{j}}=\frac{1}{\left|\omega_{j}\right|} \int_{\omega_{j}} v .
$$

Let $\left\{\Phi_{j}\right\}_{j=1}^{N}$ be a set of bounded coarse basis functions that form a partition of unity, except in a boundary layer of width $\mathcal{O}(H)$ near $\partial \Omega$. Since each support $\omega_{j} \subset \Omega_{k}$, for some $k$, the supports have finite overlap. $C_{1}$ and $C_{2}$ can now be bounded independent of the contrast in $\alpha$, if either

$$
\gamma_{2}\left(\alpha,\left\{\Phi_{j}\right\}\right)=\max _{j=1}^{N} H_{j}^{2-d}\left\|\Phi_{j}\right\|_{a}^{2} \quad \text { and } \quad \gamma_{\infty}\left(\alpha,\left\{\chi_{k}\right\}\right)=\max _{k=1}^{K} \delta_{k}^{2}\left\|\alpha^{1 / 2} \nabla \chi_{k}\right\|_{\infty}^{2}
$$

(the so-called coarse space and partitioning robustness indicators) can be bounded independent of $\alpha$, for some choice of the partition of unity $\left\{\chi_{k}\right\}_{k=1}^{K}$ subordinate to $\left\{\Omega_{k}\right\}_{k=1}^{K}$ (cf. [8]), or if $\gamma_{\infty}\left(\alpha,\left\{\Phi_{j}\right\}\right)$ can be bounded independent of $\alpha$ (cf. [17]). As mentioned above, this leads to the aim to construct coarse basis functions with minimal or bounded energy. It is also at the heart of matrix-dependent prolongation operators in multigrid methods. 
For certain binary coefficient distributions, e.g. for high-permeability inclusions in a low-permeability medium as depicted in Fig. 1(g), it was then possible in [8] to show (rigorously) under the assumption $\alpha \gtrsim 1$ that multiscale FEs (w.r.t. some coarse mesh $\mathcal{T}_{H}$ ) can provide such a basis $\left\{\Phi_{j}\right\}$, and that the indicators can be bounded independent of the contrast in $\alpha$. However, they depend on $H / \varepsilon$, where $\varepsilon$ is the minimum width of any island/gap.

Similarly, it was possible in [17] to show (again assuming $\alpha \gtrsim 1$ ) that aggregation based on a strong connection criterion (originally designed for AMG methods) leads to a coarse basis $\left\{\Phi_{j}\right\}$ for which the robustness indicators can be bounded independent of the contrast in $\alpha$. Here the bounds depend on $H / h$, since the overlap between any two supports is only $\mathcal{O}(h)$.

However, this approach to analyse robustness fails even for the simpler, reverse situation of a high-permeability medium with low-permeability inclusions (e.g. Fig. 1(f)), since in this case $\gamma_{2}\left(\alpha,\left\{\Phi_{j}\right\}\right)$ and $\gamma_{\infty}\left(\alpha,\left\{\Phi_{j}\right\}\right)$ depend on the contrast in $\alpha$ for any choice of $\left\{\Phi_{j}\right\}$. Clearly a different quasi-interpolant $\Pi$ is needed in general.

\subsection{Weighted quasi-interpolant $\mathscr{G}$ Poincaré's inequality}

The next approach to try to prove the assumptions in Theorem 1 makes use of the weighted quasi-interpolant

$$
\Pi v=\sum_{j=1}^{N} \bar{v}_{\omega_{j}}^{\alpha} \Phi_{j}, \quad \text { where } \quad \bar{v}_{\omega_{j}}^{\alpha}=\int_{\omega_{j}} \alpha v / \int_{\omega_{j}} \alpha .
$$

We describe this approach for one of the simplest coarse spaces, the piecewise linear one. The following is taken from [18] (see also [6] for earlier results). Let $V_{H}$ be the continuous, piecewise linear FE space associated with a shaperegular simplicial triangulation $\mathcal{T}_{H}$ of $\Omega$, such that $\mathcal{T}_{h}$ is a refinement of $\mathcal{T}_{H}$. The functions $\left\{\Phi_{j}\right\}_{j=1}^{N}$ are the standard nodal basis for $V_{H}$. For simplicity, we assume that $\left\{\Omega_{k}\right\}_{k=1}^{K}=\left\{\omega_{j}\right\}_{j=1}^{N}$, and choose $\chi_{k}=\Phi_{k}$ (suitably modified near $\partial \Omega$ ), so that the assumptions on $\left\{\chi_{k}\right\}$ are satisfied with $\delta_{k} \sim H_{k}$.

The key observation in [18] is now that one further assumption suffices to fully describe the dependency of the constants $C_{1}$ and $C_{2}$ in (3) on $\alpha$ :

Assumption 1. Let $\omega_{T}=\bigcup_{\left\{k: \omega_{k} \cap T \neq \emptyset\right\}} \omega_{k}$ and $H_{T}=\operatorname{diam}\left(\omega_{T}\right)$, for $T \in \mathcal{T}_{H}$, and assume that there exists a $C_{T}^{*}>0$ such that, for all $v \in V_{h}$, either

$$
\begin{gathered}
\inf _{c \in \mathbb{R}} \int_{\omega_{T}} \alpha(v-c)^{2} d \mathbf{x} \lesssim C_{T}^{*} H_{T}^{2} \int_{\omega_{T}} \alpha|\nabla v|^{2} d \mathbf{x}, \quad \text { or } \\
\partial \omega_{T} \cap \partial \Omega \neq \emptyset \text { and } \int_{\omega_{T}} \alpha v^{2} d \mathbf{x} \lesssim C_{T}^{*} H_{T}^{2} \int_{\omega_{T}} \alpha|\nabla v|^{2} d \mathbf{x} .
\end{gathered}
$$

Proposition 1. Let Assumption 1 hold. Then $C_{1}+C_{2} \lesssim C^{*}=\max _{T \in \mathcal{T}_{H}} C_{T}^{*}$. 
Proof. Let $v \in V_{h}$ and $v_{0}=\sum_{j=1}^{N} \bar{v}_{\omega_{j}}^{\alpha} \Phi_{j}$. By the Cauchy-Schwarz inequality we have $\left|\bar{v}_{\omega_{j}}^{\alpha}\right|^{2} \leq \int_{\omega_{j}} \alpha v^{2} / \int_{\omega_{j}} \alpha$, and so, using the fact that $\Phi_{j} \leq 1$,

$$
\int_{T} \alpha v_{0}^{2} \leq \sum_{j: \omega_{j} \cap T \neq \emptyset} \frac{\int_{\omega_{j}} \alpha v^{2}}{\int_{\omega_{j}} \alpha} \int_{T} \alpha \Phi_{j}^{2} \leq \int_{\omega_{T}} \alpha v^{2},
$$

which also implies $\int_{T} \alpha\left(v-v_{0}\right)^{2} \lesssim \int_{\omega_{T}} \alpha v^{2}$. Now, multiplying the left hand side by $\left|\nabla \chi_{k}\right|_{T}^{2}$ (which is a constant $\sim H_{T}^{-2}$ ) and summing over $k \geq 1$, we get

$$
\sum_{k=1}^{K}\left\|\left(v-v_{0}\right) \nabla \chi_{k}\right\|_{0, \alpha, T}^{2} \lesssim H_{T}^{-2} \int_{\omega_{T}} \alpha v^{2} .
$$

If $\left\{\Phi_{j}\right\}$ forms a partition of unity on all of $\omega_{T}$ (i.e. if $\partial \omega_{T} \cap \partial \Omega=\emptyset$ ), we can replace $v$ in (6) by $\hat{v}=v-c$, for any $c \in \mathbb{R}$, without changing the integral on the left hand side. Otherwise we set $\hat{v}=v$. In both cases, by Assumption 1

$$
\int_{\omega_{T}} \alpha \hat{v}^{2} \lesssim C_{T}^{*} H_{T}^{2} \int_{\omega_{T}} \alpha|\nabla v|^{2}
$$

Combining (6) and (7) and summing over all $T \in \mathcal{T}_{H}$ gives the bound for $C_{2}$. The bound for $C_{1}$ can be established in a similar way (cf. [18, Lem. 4.1]).

Assumption 1 postulates the existence of a discrete weighted Poincaré/ Friedrichs-type inequality on each $\omega_{T}$. It always holds, but in general the constants $C_{T}^{*}$ will not be independent of $\left.\alpha\right|_{\omega_{T}}$ and $H_{T} / h$. As described in detail in $[18, \S 3]$ (see also $[14,15,13]$ ), to obtain independence of $\alpha$, we require a certain local quasi-monotonicity of $\alpha$ on each of the regions $\omega_{T}$.

Weighted Poincaré Inequalities. Let us consider a generic coarse element $T \in \mathcal{T}_{H}$ and define the following subsets of $\omega_{T}$ where $\alpha$ is constant:

$$
\omega^{m}=\omega_{T} \cap \mathcal{Y}_{m}, \quad m=1, \ldots, M .
$$

By $\mathcal{I}_{T} \subset\{1, \ldots, M\}$ we denote the index set of all regions $\omega^{m}$ that are nonempty. Let us assume w.l.o.g. that each of these subregions is connected. We generalise now the notion of quasi-monotonicity coined in [3] by considering the following three (two) directed combinatorial graphs $\mathcal{G}^{(k)}=\left(\mathcal{N}, \mathcal{E}^{(k)}\right)$, $0 \leq k \leq d-1$, where $\mathcal{N}=\left\{\omega^{m}: m \in \mathcal{I}_{T}\right\}$ and the edges are ordered pairs of vertices. We distinguish between three (two) different types of connections.

Definition 1. Suppose that $\gamma^{m, m_{2}}=\bar{\omega}^{m} \cap \bar{\omega}^{m_{2}}$ is a non-empty manifold of dimension $k$, for $0 \leq k \leq d-1$. The ordered pair $\left(\omega^{m}, \omega^{m_{2}}\right)$ is an edge in $\mathcal{E}^{(k)}$, if and only if $\alpha_{m} \lesssim \alpha_{m_{2}}$. The edges in $\mathcal{E}^{(k)}$ are said to be of type-k.

In addition, for $1 \leq k \leq d-1$, we assume that

- $\operatorname{meas}\left(\gamma^{m, m_{2}}\right) \sim \operatorname{meas}\left(\omega^{m} \cup \omega^{m_{2}}\right)^{k / d}$, and

- $\gamma^{m, m_{2}}$ is sufficiently regular, i.e. it is a finite union of shape-regular $k$ dimensional simplices of diameter $\sim \operatorname{meas}\left(\gamma^{m, m_{2}}\right)^{1 / k}$. 
Quasi-monotonicity is related to the connectivity in $\mathcal{G}^{(k)}$. Let $m_{*} \in \mathcal{I}_{T}$ be the index of the region $\omega^{m_{*}}$ with the largest coefficient: $\alpha_{m_{*}}=\max _{m \in \mathcal{I}_{T}} \alpha_{m}$.

Definition 2. The coefficient $\alpha$ is type- $k$ quasi-monotone on $\omega_{T}$, if there is a path in $\mathcal{G}^{(k)}$ from any vertex $\omega^{m}$ to $\omega^{m *}$.

The following lemma summarises the results in $[14,15,13]$. The existence of a benign constant $C_{T}^{*}$ that is independent of $\alpha$ is directly linked to quasimonotonicity, the way in which $C_{T}^{*}$ depends on $H_{T} / h$ to the type.

Lemma 1. Let $\omega_{T} \subset \mathbb{R}^{d}, d=2,3$. If $\alpha$ is type- $k$ quasi-monotone on $\omega_{T}$, then (4) holds with

$$
C_{T}^{*}= \begin{cases}1, & \text { if } k=d-1, \\ 1+\log \left(\frac{H_{T}}{h}\right), & \text { if } k=d-2, \\ \frac{H_{T}}{h}, & \text { if } k=d-3 .\end{cases}
$$

A similar result can also be established in the case where $\partial \omega_{K} \cap \partial \Omega \neq \emptyset$, i.e. the case of Friedrichs inequality (5), see e.g. [18, §3] for details.

Quasi-monotonicity is crucial. If the coefficient is not quasi-monotone, e.g. the situation in Figure 1(d), then $C^{*}$ cannot be bounded independent of $\alpha$. See [18, Ex. 3.1] for a counter example. If the coarse mesh is not adjusted in certain critical areas of $\Omega$, then $V_{H}$ is in general not robust. The numerical results in [18] show that this is indeed the case and that quasi-monotonicity is necessary and sufficient. However, a few simple adjustments suffice, namely $\mathcal{T}_{H}$ has to be sufficiently fine in certain "critical" areas of $\Omega$ :

1. Choose $H_{T} \leq \varepsilon_{m}$, for all $T \in \mathcal{T}_{H}$ that intersect a region $\mathcal{Y}_{m}$ that is bordered by two regions $\mathcal{Y}_{m^{\prime}}$ and $\mathcal{Y}_{m^{\prime \prime}}$ with $\alpha_{m^{\prime}} \gg \alpha_{m}$ and $\alpha_{m^{\prime \prime}} \gg \alpha_{m}$. Here $\varepsilon_{m}$ denotes the width of $\mathcal{Y}_{m}$ at its narrowest point. This ensures that $\alpha$ is quasi-monotone on all regions $\omega_{T}$ that intersect $\mathcal{Y}_{m}$.

2 . Choose $H_{T} \lesssim h$, near any point or edge where $\alpha$ is only type- $(d-2)$ or type- $(d-3)$ quasi-monotone, i.e. near any cross point.

Usually a logarithmic growth $C^{*} \sim \max _{T} \log \left(H_{T} / h\right)$ is acceptable, and so even regions where the coefficient is type- $(d-2)$ quasi-monotone do not require any particular attention.

For an arbitrary piecewise constant coefficient function $\alpha$ there will often only be a relatively small (fixed) number of regions $\omega_{T}$ where $\alpha$ is not quasi-monotone (see e.g. Figures 1(b-e)). Therefore it is very easy to ensure through some local refinement of $\mathcal{T}_{H}$ near these regions that $C^{*} \sim 1$ (or $\left.C^{*} \sim \log (H / h)\right)$. Note that crucially, this local refinement does not mean that $\mathcal{T}_{H}$ has to be aligned with coefficient jumps anywhere in $\Omega$. The coarse grid merely has to be sufficiently fine in regions where $\alpha$ is not quasi-monotone. Ideas on how to adapt $\mathcal{T}_{H}$ in such a way are suggested in [18].

"Exotic" coarse spaces. Substructuring-type ("exotic") coarse spaces (as suggested in $[4,3,16])$ can be analysed in a similar way. Here the coarse basis functions are constructed as $a$-harmonic extensions of face, edge or vertex "cut" functions associated with a non-overlapping decomposition $\mathcal{T}_{H}$ of the 
domain. This decomposition may be related to the overlapping partitioning $\left\{\Omega_{k}\right\}$, or it may come from a separate coarse grid (not necessarily simplicial). If the coefficient does not vary along any of the edges/faces of $\mathcal{T}_{H}$, then the space can be analysed like the piecewise linear one above, using in addition the energy minimising property of the $a$-harmonic extension (cf. [14]). If the coefficient does vary along an edge/face, then special weighted Poincaré inequalities for functions with vanishing weighted averages across edges/faces are required. These have recently been introduced in the context of FETI-DP methods in [12], which also analyses the robustness of the "cut" functions. An explicit analysis in the context of overlapping Schwarz does not yet exist.

\subsection{Abstract minimisation with functional constraints}

An alternative to refining the coarse mesh in regions where $\alpha$ is not type$(d-1)$ or type $-(d-2)$ quasi-monotone, is to associate more than one basis function (with possibly identical supports) with each subdomain $\Omega_{k}$. Let

$$
V_{0}=\operatorname{span}\left\{\Phi_{k, j}=I_{h}\left(\chi_{k} \Psi_{k, j}\right): j=1, \ldots, N_{k}, \quad k=1, \ldots, K\right\},
$$

where $\Psi_{k, j}, j=1, \ldots, N_{k}$, are suitable FE functions in $V_{h}\left(\bar{\Omega}_{k}\right)$ (that do not vanish on $\partial \Omega_{k}$ ) such that the functions $\left\{\Phi_{k, j}\right\} \subset V_{h}$ are linearly independent. Good choices for the functions $\Psi_{k, j}$ are the lowest modes of local eigenproblems, or more generally, energy minimising functions that satisfy suitable constraints. The following analysis is from [19] (see [7, 2] for related work).

In particular, let us assume that, for every $\Omega_{k}$, we have a collection of linear functionals $\left\{f_{k, j}\right\}_{j=1}^{N_{k}} \subset V_{h}\left(\bar{\Omega}_{k}\right)^{\prime}$ and let

$$
\Psi_{k, j}=\underset{v \in V_{h}\left(\bar{\Omega}_{k}\right)}{\arg \min }|v|_{a}^{2}, \quad \text { subject to } \quad f_{k, l}\left(\Psi_{k, j}\right)=\delta_{j l} \quad j, l=1, \ldots, N_{k} .
$$

Now, for any $v \in V_{h}$, choose the following quasi-interpolant

$$
\Pi v=\sum_{k=1}^{K} I_{h}\left(\chi_{k} \Pi_{\Omega_{k}} v\right), \quad \text { where } \quad \Pi_{\Omega_{k}} v=\sum_{j=1}^{N_{k}} f_{k, j}\left(\left.v\right|_{\Omega_{k}}\right) \Psi_{k, j},
$$

i.e. a linear combination of the basis functions $\Phi_{k, j}$ with weights $f_{k, j}\left(\left.v\right|_{\Omega_{k}}\right)$. Then the bounds on $C_{1}$ and $C_{2}$ in Theorem 3 depend only on the stability and on the local $L_{2}$-approximation properties of $\Pi_{\Omega_{k}}$ on each $\Omega_{k}$.

Theorem 2. For all $k=1, \ldots, K$ and for all $v \in V_{h}\left(\bar{\Omega}_{k}\right)$, let

$$
\left\|\Pi_{\Omega_{k}} v\right\|_{a, \Omega_{k}}^{2} \leq\|v\|_{a, \Omega_{k}}^{2} \quad \text { and }\left\|v-\Pi_{\Omega_{k}} v\right\|_{0, \alpha, \Omega_{k}}^{2} \lesssim \operatorname{diam}\left(\Omega_{k}\right)^{2}\|u\|_{a, \Omega_{k}}^{2} .
$$

Then $C_{1}=\mathcal{O}(1)$ and $C_{2} \lesssim\left(\operatorname{diam}\left(\Omega_{k}\right) / \delta_{k}\right)^{2}$.

Proof. See [19, Thm. 5.1]. 
Note that the minimisation problems in (9) are local to each subdomain. There are suitable choices for the functionals $f_{k, j}$ that guarantee (10) and that lead to practical algorithms to construct the functions $\Psi_{k, j}, j=1, \ldots, N_{k}$ :

- $f_{k, j}(v)=\left(\Psi_{k, j}, v\right)_{0, \alpha, \Omega_{k}}$ where $\Psi_{k, j}$ is the $j$ th eigenfunction corresponding to the variational eigenproblem: Find $\eta \in V_{h}\left(\bar{\Omega}_{k}\right)$ and $\lambda \geq 0$, such that

$$
a(\eta, w)=\lambda(\eta, w)_{0, \alpha, \Omega_{k}}, \quad \text { for all } \quad w \in V_{h}\left(\bar{\Omega}_{k}\right) .
$$

This has first been suggested and analysed in [7].

- $f_{k, j}(v)=\left(\Psi_{k, j}, v\right)_{0, \alpha, \partial \Omega_{k}}$ where $\Psi_{k, j}$ is the $j$ th eigenfunction corresponding to a variational eigenproblem similar to (11), but with $(\eta, w)_{0, \alpha, \partial \Omega_{k}}$ instead of $(\eta, w)_{0, \alpha, \Omega_{k}}$ on the right hand side of (11), i.e. an eigenproblem of Steklov-Poincaré type. This has been analysed in [2].

- $f_{k, j}(v)=\bar{v}_{D_{k, j}}^{\alpha}$ where $\left\{D_{k, j}\right\}_{j=1}^{N_{k}}$ is a suitable non-overlapping partitioning of $\Omega_{k}$ such that the weighted Poincaré inequality (4) holds on each $D_{k, j}$ (e.g. $D_{k, j}=\Omega_{k} \cap \mathcal{Y}_{j}$ ). The construction of $\left\{\Psi_{k, j}\right\}$ requires the solution of $N_{k}$ local saddle point systems and was suggested and analysed in [19].

It has been shown in [7, 2] how (10) can be proved (directly) in the first two cases, essentially based on the observation that the coarse space consists of the lowest modes corresponding to the operator pencil associated to the energy and to the weighted $L_{2}$-norm. But the assumptions can be proved for a much wider class of functionals using the following abstract approximation result in [19]. This result is related to the classical Bramble-Hilbert lemma.

Abstract Approximation Result. Consider an abstract symmetric and continuous bilinear form $a(\cdot, \cdot): V \times V \mapsto \mathbb{R}$, as well as a collection of linear functionals $\left\{f_{l}\right\}_{l=1}^{m} \subset V^{\prime}$, where $V \subset \mathcal{H}$ and $\mathcal{H}$ is a Hilbert space with norm $\|\cdot\|$. We make the following assumptions on $a(\cdot, \cdot), V, \mathcal{H},\|\cdot\|$ and $\left\{f_{l}\right\}$ :

A1. $a(\cdot, \cdot)$ is positive semi-definite and defines a semi-norm $|\cdot|_{a}$ on $V$, i.e.

$$
|v|_{a}^{2}=a(v, v) \geq 0, \quad \text { for all } \quad v \in V .
$$

In addition, for $v \in V$, the expression $\sqrt{\|v\|^{2}+|v|_{a}^{2}}$ defines a norm on $V$. A2. Let $c_{q}$ be a generic constant. For all $\mathbf{q} \in \mathbb{R}^{m}$ there exists a $v_{\mathbf{q}} \in V$ with

$$
f_{l}\left(v_{\mathbf{q}}\right)=q_{l}, \quad \text { and } \quad\left\|v_{\mathbf{q}}\right\| \lesssim c_{q}\|\mathbf{q}\|_{l^{2}\left(\mathbb{R}^{m}\right)} .
$$

A3. There are two constants $c_{a}$ and $c_{f}$ such that

$$
\|v\|^{2} \leq c_{a}|v|_{a}^{2}+c_{f} \sum_{l=1}^{m}\left|f_{l}(v)\right|^{2}, \quad \text { for all } v \in V .
$$

Now, as in the specific case above, define for all $v \in V$,

$$
\pi v=\sum_{l=1}^{m} f_{l}(v) \psi_{l}, \quad \text { where } \quad \psi_{l}=\arg \min _{v \in V}|v|_{a}^{2}, \quad \text { subject to } \quad f_{l}\left(\psi_{j}\right)=\delta_{j l} .
$$


Then the following inequalities hold; see [19, Thm. 3.3].

Theorem 3. Let Assumptions A1-3 be satisfied. Then, for all $u \in V$ :

$$
|\pi u|_{a} \leq|u|_{a} \quad \text { and } \quad\|u-\pi u\| \leq \sqrt{c_{a}}|u|_{a} .
$$

(Note that they are independent of the constants $c_{q}$ and $c_{f}$ in $\mathbf{A 2}$ and A3.)

In the specific case considered above, on an arbitrary subdomain $\Omega_{k}$, Assumption A1 is naturally satisfied with $\mathcal{H}=L_{2}\left(\Omega_{k}\right)$ and $\|\cdot\|=\|\cdot\|_{0, \alpha, \Omega_{k}}$. Assumption A2 merely ensures that the linear functionals are linearly independent. Thus, the question of coarse space robustness is reduced to verifying Assumption A3. For one functional, i.e. for $m=1$, this reduces to the weighted Poincaré inequality in Section 3.2 and to the restrictions on the coefficients made there. For more than one functional, it opens the possibility to get coefficient robustness even in the case of non-quasi-monotone coefficients, such as those depicted in Figures 1(b-d) and even (h). See [19, 7, 2] for the complete analysis and some numerical experiments that confirm the robustness for the functionals defined on the previous page. See also [20] for a more recent extension to systems of elliptic PDEs (such as linear elasticity).

\section{References}

[1] J.H. Bramble and J. Xu. Some estimates for a weighted L2 projection. Math. Comput., 56:436-476, 1991.

[2] V. Dolean, F. Nataf, R. Scheichl, and N. Spillane. Analysis of a two-level Schwarz method with coarse spaces based on local Dirichlet-to-Neumann maps. Technical Report HAL-00586246, Hyper Articles en Ligne, 2011.

[3] M. Dryja, M. Sarkis, and O.B. Widlund. Multilevel Schwarz methods for elliptic problems with discontinuous coefficients in three dimensions. Numer. Math., 72:313-348, 1996.

[4] M. Dryja, B.F. Smith, and O.B. Widlund. Schwarz analysis of iterative substructuring algorithms for elliptic problems in three dimensions. SIAM J Numer. Anal., 31:1662-1694, 1994.

[5] Y. Efendiev, J. Galvis, R. Lazarov, and J. Willems. Robust domain decomposition preconditioners for abstract symmetric positive definite bilinear forms. Technical Report 2011-05, RICAM, Linz, 2011.

[6] J. Galvis and Y. Efendiev. Domain decomposition preconditioners for multiscale flows in high contrast media. Multiscale Model. Sim., 8(4):1461-1483, 2010.

[7] J. Galvis and Y. Efendiev. Domain decomposition preconditioners for multiscale flows in high contrast media: reduced dimension coarse spaces. Multiscale Model. Sim., 8(5):1621-1644, 2010.

[8] I.G. Graham, P. Lechner, and R. Scheichl. Domain decomposition for multiscale PDEs. Numer. Math., 106:589-626, 2007. 
[9] I.G. Graham and R. Scheichl. Robust domain decomposition algorithms for multiscale PDEs. Numer. Meth. Part. D. E., 23:859-878, 2007.

[10] J. Mandel and M. Brezina. Balancing domain decomposition for problems with large jumps in coefficients. Math. Comp., 65:1387-1401, 1996.

[11] J. Mandel, M. Brezina, and P. Vanek. Energy optimisation of algebraic multigrid bases. Computing, 62:205-228, 1999.

[12] C. Pechstein, M. Sarkis, and R. Scheichl. New theoretical coefficient robustness results for FETI-DP. Submitted Proc DD20 San Diego, 2011.

[13] C. Pechstein and R. Scheichl. Weighted Poincaré inequalities. NuMaReport 2010-10, Inst. Comput. Mathematics, J.K. University Linz, 2010.

[14] C. Pechstein and R. Scheichl. Analysis of FETI methods for multiscale PDEs - part II: Interface variation. Numer. Math., 118(3):485-529, 2011.

[15] C. Pechstein and R. Scheichl. Weighted Poincaré inequalities and applications in domain decomposition. In Y. Huang et al., editors, Domain Decomposition Methods in Science and Engineering XIX, volume 78 of Lecture Notes in Computational Science and Engineering, 2011.

[16] M. Sarkis. Nonstandard coarse spaces and Schwarz methods for elliptic problems with discontinuous coefficients using non-conforming elements. Numer. Math., 77:383-406, 1997.

[17] R. Scheichl and E. Vainikko. Additive Schwarz and aggregation-based coarsening for elliptic problems with highly variable coefficients. Computing, 80:319-343, 2007.

[18] R. Scheichl, P.S. Vassilevski, and L. Zikatanov. Multilevel methods for elliptic problems with highly varying coefficients on non-aligned coarse grids. Report LLNL-JRNL-451252, Lawrence Livermore Nat. Lab., 2010.

[19] R. Scheichl, P.S. Vassilevski, and L. Zikatanov. Weak approximation properties of elliptic projections with functional constraints. Multiscale Model. Simul., 2011. To appear.

[20] N. Spillane, V. Dolean, P. Hauret, F. Nataf, C. Pechstein, and R. Scheichl. Abstract robust coarse spaces for systems of PDEs via generalized eigenproblems in the overlaps. Technical report, 2011. submitted.

[21] A. Toselli and O. Widlund. Domain Decomposition Methods - Algorithms and Theory. Springer, Berlin, 2005.

[22] P.S. Vassilevski. Multilevel Block-Factorization Preconditioners: Matrixbased Analysis and Algorithms for Solving FE Equations. Springer, 2008.

[23] J. Xu. Iterative methods by space decomposition and subspace correction. SIAM Review, 34:581-613, 1992.

[24] J. Xu and Y. Zhu. Uniform convergent multigrid methods for elliptic problems with strongly discontinuous coefficients. Math. Mod. Meth. Appl. S., 18:1-29, 2008.

[25] J. Xu and J. Zou. Some nonoverlapping domain decomposition methods. SIAM Review, 40:857-914, 1998. 